\title{
SÍNTESE DE DERIVADOS DO GLICEROL: UMA AÇÃO PARA VALORIZAR O PRINCIPAL REJEITO DA INDÚSTRIA DE BIODIESEL
}

\author{
Autores: *COSTA, A. K. de M. (IFRN) SILVA, F. F. M. da (IFRN) PAIVA, L. M. M. de (IFRN)
}

\section{RESUMO}

A produção de biodiesel no Brasil têm crescido significativamente nos últimos anos, gerando um considerável volume de glicerol como subproduto do processo. Com isso, a produção de derivados do glicerol tem um papel importante na consolidação da indústria de biocombustíveis (biodiesel), uma vez que agrega valor ao seu principal subproduto (glicerol). Desta forma, este estudo visa demonstrar o processo de produção de derivados do glicerol por via química.
Foram produzidos dois derivados, o cetal originado da reação do glicerol com a acetona, e o cetal acetilado com um excelente rendimento de $90 \%$. Ambos os produtos obtidos foram caracterizados por cromatografia em camada delgada e através de cromatografia gasosa acoplada a espectrometria de massas (CG-MS), confirmando os produtos esperados.

PALAVRAS-CHAVE: Biodiesel, Glicerol, Derivados do glicerol

\section{SYNTHESIZING GLYCEROL DERIVATIVES: RAISING THE VALUE OF BIODIESEL WASTE}

\begin{abstract}
Biodiesel production in Brazil have grown significantly in recent years, generating a considerable volume of glycerol, byproduct of the process. The production of derivatives of glycerol has an important role in the consolidation of the biofuels industry (thought biodiesel), as it adds value to its primary byproduct (glycerol). Thus, this study is to demonstrate the production process of
\end{abstract}

glycerol derivatives by chemical means. derivatives of glycerol production process by chemical means. Two derivatives were produced, the ketal of glycerol originated reaction with acetone ketal and acetylated with an excellent yield of $90 \%$. Both products were characterized by thin layer chromatography and by gas chromatography-mass spectrometry (GC-MS), confirming the expected products.

KEY-WORDS: Biodiesel, Glycerol, Derivatives of glycerol 


\section{SÍNTESE DE DERIVADOS DO GLICEROL: UMA AÇÃO PARA VALORIZAR O PRINCIPAL REJEITO DA INDÚSTRIA DE BIODIESEL}

\section{INTRODUÇÃO}

A crescente produção de biodiesel nos últimos anos traz consigo a geração de coprodutos, tendo como principal o glicerol, que constitue aproximadamente $10 \%$ da massa total do produto. O glicerol é um tri-álcool que tem como características líquido incolor, inodoro, viscoso, higroscópico e de sabor adocicado (MOTA, SILVA, GONÇALVES, 2009).

A produção em massa de biodiesel ocasionou uma queda gradativa no custo da glicerina. Nesse contexto, a síntese de derivados do glicerol fortalece a produção de biodiesel, uma vez que esta agrega valor ao principal rejeito gerado no processo. Esses derivados (cetais, ésteres) possuem aplicações em diversas áreas (farmacêutica, automotiva, cosmética, etc), sendo uma alternativa de aproveitamento do grande volume de glicerol (MOTA, SILVA, GONÇALVES, 2009).

Com isso, o presente trabalho teve como objetivo demonstrar o processo de produção de derivados do glicerol a fim de contribuir para dispersão do conhecimento e, consequentemente, fortalecer as indústrias de biodiesel no Brasil.

\section{MATERIAIS E MÉTODOS}

\subsection{PROCEDIMENTO DE FORMAÇÃO DO CETAL POR CATÁLISE ÁCIDA:}

A mistura de $0,5 \mathrm{~mol}(46 \mathrm{~g})$ de glicerol juntamente com $2 \mathrm{~mol}(148,72 \mathrm{~mL})$ de acetona e $1 \mathrm{~mL}$ de $\mathrm{HCl}$ concentrado foi adicionada em balão em sistema de refluxo, por 2 horas à 100 ㄷ. Em seguida a reação foi concentrada em rotaevaporador e logo após, neutralizada com solução aquosa de $\mathrm{NaHCO}_{3}$ a $5 \%$. Na sequência o meio foi colocado em funil de decantação com $10 \mathrm{~mL}$ de etanol e solução saturada de $\mathrm{NaCl}$ (sal). Foi realizada extração do meio com acetato de etila $(5 \times 20 \mathrm{~mL})$. A fase orgânica foi seca com $\mathrm{Na}_{2} \mathrm{SO}_{4} \mathrm{e}$ concentrada em rotaevaporador. $\mathrm{O}$ produto foi caracterizado por cromatografia gasosa acoplada a espectrometria de massas (CG-MS) para confirmação da obtenção do produto desejado.

\subsection{PROCEDIMENTO PARA ACETILAÇÃO DO CETAL}

A mistura de 0,01 mol de cetal juntamente com 0,03 mol de anidrido acético e 0,01 mol de piridina sob agitação magnética à temperatura ambiente por 4 horas. Em seguida, foi adicionado $20 \mathrm{~mL}$ de acetato de etila e realizada extração com solução aquosa de $\mathrm{CuSO}_{4} \cdot \mathrm{H}_{2} \mathrm{O}(20 \% \mathrm{~m} / \mathrm{V}, 4 \times 20 \mathrm{~mL})$. Por fim, secou-se a fase orgânica da reação com $\mathrm{Na}_{2} \mathrm{SO}_{4} \mathrm{e}$ concentrou-se em rotaevaporador.

\section{RESULTADOS E DISCUSSÕES}

Após a síntese do cetal e acetilação do mesmo, estes foram caracterizados através de CG-MS (cromatografia gasosa acoplada a espectrometria de massas) resultando no espectro apresentado na Figura 01, onde pode-se observar que o cetal foi identificado com tempo de retenção em 6,9 minutos (A) e acetilado em 8,7minutos (B).

Figura 01 - espectro de massa do cetal (A) e cetal acetilado (B) 

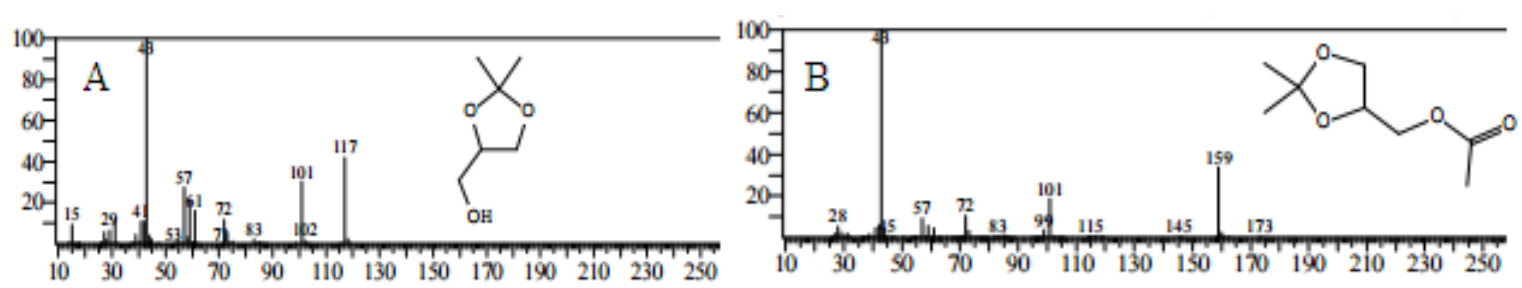

FONTE: Elaborado pelo autor

A metodologia adotada foi comparada com dados da literatura. A partir de tais pesquisas pôde-se perceber que o rendimento obtido do cetal, $21,21 \%$, não foi satisfatório, pois Mota et al. (2012) e Dantas (2013), que realizaram reações a partir do glicerol com acetona obtiveram rendimentos acima de $70 \%$ e $52 \%$, respectivamente. Já o cetal acetilado apresentou rendimento de 90\%. Nas Figuras 02 e 03 abaixo, estão dispostas as reações químicas de formação do cetal e cetal acetilado e seus respectivos rendimentos.

Figura 02 - Reação química de formação do cetal

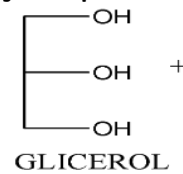<smiles>CC(=O)ON=NOC(C)=O</smiles>

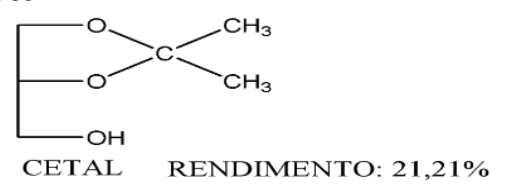

FONTE: Elaborado pelo autor

Figura 03 - Reação química de formação do cetal acetilado

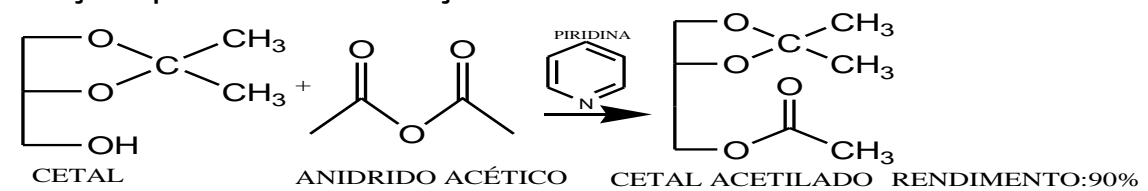

FONTE: Elaborado pelo autor

\section{CONCLUSÃO}

Os resultados obtidos nesta pesquisa mostram que é possível a produção de derivados do glicerol, contudo novos métodos estão sendo estudados para se obter um melhor rendimento na produção do cetal. O glicerol é uma matéria - prima versátil, barata e adequada ambientalmente e economicamente que dispõe de uma variedade de aplicações, podendo potencializar o seu valor com a produção de derivados e fortalecendo também as indústrias de biodiesel.

\section{AGRADECIMENTOS}

Os autores agradecem ao Instituto Federal de Educação, Ciência e Tecnologia do Rio Grande do Norte - IFRN, Campus Apodi, ao CNPq pelas bolsas concedidas e apoio financeiro para realização do projeto.

\section{REFERÊNCIAS}

DANTAS, P. R. de O. Obtenção de derivados do glicerol utilizando enzimas da casca de laranja como catalisador. Instituto Federal de Educação, Ciência e Tecnologia do Rio Grande do Norte (IFRN), 2013.

MOTA, C. J. A.; SILVA, C. X. A.; GONÇALVES, V. L. C. Gliceroquímica: novos produtos e processos a partir da glicerina de produção de biodiesel. Quim. Nova, Rio de janeiro, vol. 32, n. 3, p. 639-648, 2009.

MOTA, M. B. S.; MOTA, C. J. A.; OZORIO, L. P.; PIANZOLLI, R. Reactivity of glycerol/acetone ketal (solketal) and glycerol/formaldehyde acetals toward acidcatalyzed hydrolysis. Escola de Química, Universidade Federal do Rio de Janeiro (UFRJ), 2012. 